\title{
TEACHING ON CHINESE WRITING IN BINUS UNIVERSITY 印尼建国大学中文写作教学
}

\author{
Ma Feng（马峰） \\ Chinese Departement, Faculty of Languange and Culture, Binus University \\ Jl. Kemanggisan Ilir III no.45, Kemanggisan/ Palmerah, Jakarta Barat 11480 \\ mafengde_2009@hotmail.com
}

\begin{abstract}
Combination of practice teaching Chinese as a foreign language in BINUS University, this article takes the Chinese writing teaching as an example, and analyses "as the student core, as the fun concept" writing modes. Firstly, serious explain: increasing the vitality of classroom teaching, using multimedia methods to make students get interested in; Secondly, article practice: making the written expression be the basis and revealing true feelings as commander, constantly pursue the aesthetic article; Thirdly, feedback and communion: using Facebook, Binusmaya, Binusblog, Chinese newspapers and other channels of interaction to communicate between teachers and students. Through the three steps, we could select the theme which students loved, create a relaxed atmosphere in the writing class, and then the students will be pleasure of writing gradually.
\end{abstract}

Keywords: writing, BINUS University, classroom teaching

\section{内容摘要}

结合建国大学的写作教学, 本文对 “以学生为核心, 以趣味为理念” 的写作模式进行探讨: 一、精 心讲解: 增加课堂教学的生动性, 利用多媒体等方式引导学生兴趣; 二、文章操练: 以书面表达能力为 基础，以真实情感流露为统帅，不断追求文章的艺术美感; 三、反馈交流：通过 Facebook，Binusmaya， Binusblog, 中文报纸等多种渠道师生互动。通过三层梯级深入, 选择学生喜闻乐见的题材, 为写作课营 造轻松的氛围, 进而让学生步入快乐写作的佳境。

关键词: 写作, 建国大学, 课堂教学 


\section{前言}

写作是语言学习的提升阶段, 引导学生对写作的兴趣有助于语言学习的深入。通过对印尼 建国大学中文系学生的调查显示, 由于受到影视媒体、电脑网络等因素的冲击, 目前学生的阅 读与写作兴趣普遍不高。“印尼曾经是东南亚地区华文教育相当兴旺发达的国家。但又是华人历 经磨难和坎坷的国家。1966 年, 在军人政权一纸命令下, 印尼的华文教育荡然无存, 丧失殆尽。 华文教育自此中断了 32 年。至今, 许多 50 岁以下的华人不谙华文, 令人悲愤难忍。1998 年 5 月, 苏哈托政府垮台, 印尼政府大幅度调整华人政策, 实行多元文化方针, 华文教育迎来了新 的春天，复苏振兴的势头很好。但也存在不少问题和困难。”（黄昆章，《市民日报》，2009）随 着中国经济的发展, 国际影响力的增强, 现在华文的国际地位也随之提升; 同时, 印尼开放的 文化政策以及与中国交流合作的增多，这些都有利于华文的复苏。另一方面，32 年华文教育断 层的不利影响仍然存在, 如何提高学生的华文学习兴趣, 如何提升学生的华文写作水平, 这些 都是印尼华文教育所面临的挑战。

\section{内容}

\section{一、教学对象及调查分析}

笔者的教学对象是建国大学中文系二年级下学期的两个班 (04PAL，31 人; 04PBL，28 人; 共 59 名学生), 使用的教材是北京语言大学出版社的《汉语写作 (三年级用)》。该教材编写的 初衷是为到中国学习汉语的外国留学生设计, 按年级划分成三册, 每册学习时间为一年。建国 大学的时间安排由每册一年调整为半年, 从一年级下学期开始使用, 到二年级下学期使用的教 材便是第三册。《汉语写作 (三年级用)》属于写作教学的高级阶段教材, 二年级学生在学习过 程中稍显吃力, 时间也比较紧促 (每学期 13 课时, 每课时 100 分钟), 这也是课程安排的不足 之处。笔者在教学过程中, 以该教材为依据, 适当运用多媒体课件, 结合印尼学生的实际情况 进行多渠道教学探索。融合多种资源, 以快乐写作为理念, 培养学生的学习兴趣, 充分发挥学 生的积极性, 以期达到教学效果优化。为了更深入的了解建国大学中文系学生的学习现状, 笔 者在 2009 年 5 月与 8 月对学生分别进行了两次调查。通过调查问卷, 查找出学生学习的难点, 也发现了他们学习的兴趣所在, 这有助于进行更好、更有效的教学策略应对。结合学生及教师 们的交流意见，对调查问卷进行了如下统计分析。

调查问卷一: (许丽妮、马峰, 2009:350-355)

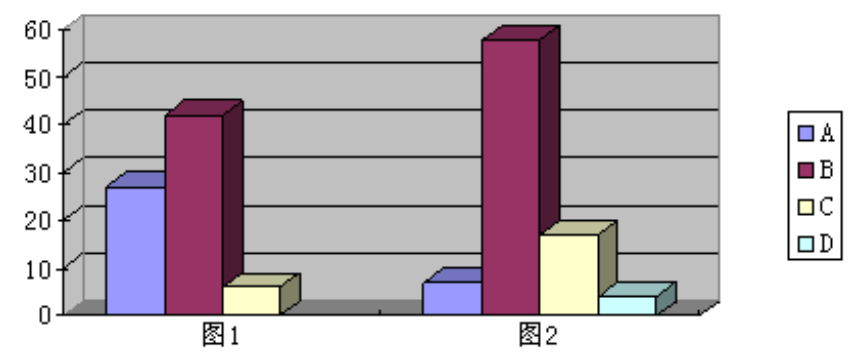

(注: 上图纵轴为人数, 横轴分别为图 1、图 2, $\mathrm{ABCD}$ 为各图所示问题选项, 各选项后的方括号内为选择人数统计。) 
图 1: 你喜欢哪种讲课方法?
A. 以课本为主 [27]
B. 使用多媒体的 Power Point [42]
C. 其他 [6]

图 2: 你最喜欢的课外学习汉语的方式?
A. 阅读报刊 [7]
B. 看华文影视 [58]
C. 听、唱华文歌 [17]
D. 其他 [4]

2009 年 5 月对建国大学中文系多媒体教学方法的使用作了一次调查。调查课程是中国历史 课和中国文学课, 调查对象是笔者授课的四个班级。调查问卷共收集 73 份, 由于学生在一题中 选择多项或没有选择答案, 所以统计结果存在一定误差, 但总体上符合调查问卷规范, 因此属 于有效调查。图 1、2 表明: 学生多喜欢教师运用 Power Point 进行授课。通过影音媒体的运用, 学生可以在轻松快乐的氛围下学习。由于学生普遍对中国文学、中国历史了解较少, 所以在学 习中感到比较困难。教师在课程讲解中运用 PowerPoint 课件, 采用图片和音乐等方式, 对学生 学习帮助较大, 有助于培养学生的学习兴趣。Power Point 在教学中的运用可以化繁为简、化 难为易, 学生对中国历史课和文学课的学习逐渐增加了兴趣。

调查问卷二:

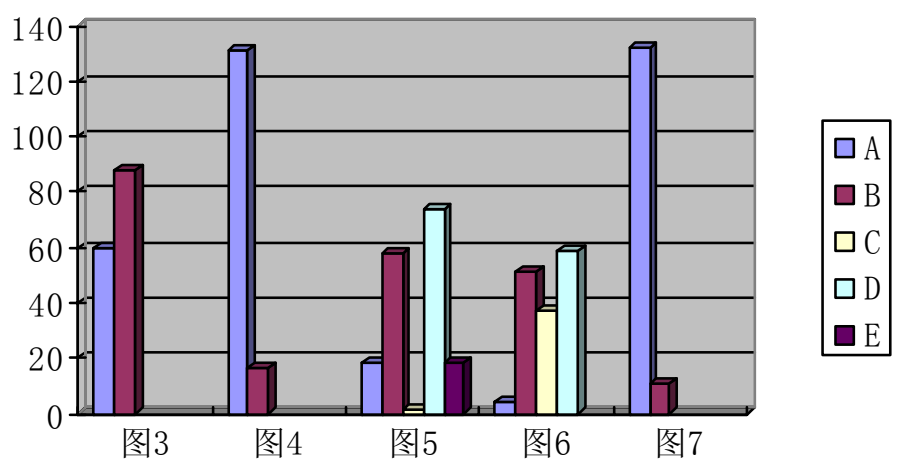

(注: 上图纵轴为人数, 横轴分别为图 3、图 4、图 5、图 6、图 7, $\mathrm{ABCDE}$ 为各图所示问题选项, 各选项后的方括号内为选择人数统计。)

图 3: 你是否喜欢写作?
A. 喜欢 [60]
B. 不喜欢 [88]

图 4: BINUS 的华文写作课对你是否有帮助?
A. 有 [131]
B. 没有 [16]

图 5: 你的业余爱好（课外时间的安排）?
A. 读书 [18]
B. 旅游 [58]
C. 写作 [1]
D. 上网 $[74]$
E. 其他 [18]

图 6: 你喜欢在哪里写文章?
A. 报纸、杂志 [4]
B. 网络 [51]
C. 写作课 [37］
D. 个人日记 [59］

图 7: 你是否发表过文章?
A. 没有 [132］
B. 有 (请写出)
[11] 
本次调查于 2009 年 8 月进行, 主要针对写作课教学。调查范围是已经修完全部写作课程的 建国大学中文系三年级、四年级学生。回收有效调查问卷 148 份。调查显示, 目前建国大学的 写作教学很不理想, 学生大多不喜欢写作。课外时间主要是上网、旅游、看电视、看电影、听 音乐等, 尤其令人震惊的是业余爱好写作的学生只有一名。学生缺乏写作动机, 写作兴趣不高, 写作数量很低。学生写的文章主要来源于写作课堂、个人日记和网络, 写作课堂则是教师安排 的写作任务, 个人日记和网络所写的文章则以简短的随笔感想为主。本次调查发现有十一名学 生曾发表过文章, 其中五名是经过写作课教师辅导后推荐发表到印尼《国际日报》、《星洲日报》。 虽然写作教学面临很多问题, 但是可以看出建国大学中文系的写作课对学生帮助较大。如何更 好的发挥写作课的作用, 如何让写作变的轻松快乐呢? “以学生为核心, 以趣味为理念” 的快 乐写作教学模式将有助于教师解决这一难题。

\section{二、快乐写作教学策略}

\section{（一） 精心讲解}

成功的教学需要教师的全身心投入, 把课堂作为激发学生兴趣的突破口。如何经营自己的 课堂, 如何让学生乐在其中, 教师必须精心策划自己的教学策略。“多媒体技术运用于对外汉语 教学, 为这一领域提供了更为广阔的思路和前景。和以往的传统教学手段相比, 多媒体手段在 许多方面显示出无以取代的优势。随着信息技术的飞速发展, 多媒体技术已广泛运用于外语教 学。它为对外汉语教学带来了新的希望和前景, 为新形势下对外汉语教学模式的变革拓宽了思 路。” (纪晓静, 2002：45-47) 笔者在教学过程中对是否使用多媒体技术做了一些试验, 结果表 明多媒体技术的运用有助于活跃课堂气氛和提高教学效果。

例析：《汉语写作 (三年级用)》第一章 “游记” 的讲解：

第一步, 精心酛酿, 热身导入。新学期开始, 学生还沉浸在假期的温馨浪漫之中, 写作第 一课的 “游记” 题材刚好可以趁热打铁, 让学生畅所欲言。教师要边听边选取关键词予以总结 列出。

第二步, 课件运用, 活跃气氛。学生讨论后, 还徘徊于视觉美景之中, 此时进入课堂的讲 解。如图 1 所示, 学生多喜欢教师使用 Power Point 课件进行授课。因此, 教师制作的精美 Power Point 课件可以给学生最直观的视觉印象, 通过自然美景、名胜古迹引发学生的心灵悸动, 让 学生有所感悟。欣赏之后, 让学生思考怎样写一篇吸引人的游记, 然后教师总结游记的写法。 此时, 学生便会感到 “山穷水复疑无路, 柳暗花明又一村”, 豁然开朗的感觉让学生的体会更加 深刻。Power Point 运用是讲解的关键, 可以让学生感到写作并不是那么枯燥艰难, 有助于调 整学生的心态, 进而提高学生的写作兴趣。

第三步, 经典赏析, 点评互动。例文《迷人的夏季牧场》简析, 学生快速汶览, 感受文章 的结构与语言。让学生选取自己最喜欢的语句段落, 同时进行身份的置换—— “假如你身处夏 季牧场, 你会有什么样的感觉？假如你写一篇夏季牧场的游记, 你会如何写呢？”学习例文的 优点, 充分发挥学生的想象。 
第四步, 拉近距离, 激发热情。结合学生的情况, 对学生们熟悉的一处印尼风景名胜进行 写作思路的介绍, 选择一篇相关游记文章进行示范。如笔者在《印尼星洲日报》旅游版的一篇 游记《秀甲天下的绿色家园一一茂物植物园游记 (Kebun Raya Bogor)》。茂物植物园毗邻印尼 总统行宫, 风景优美, 享誉海内外。很多学生曾经到茂物旅游, 贴近身边生活的文章更容易引 起学生的共鸣。如果教师有相关游记文章予以展示, 效果颇佳。经典文章往往让学生产生遥不 可及的感觉, 无形的压力会扑灭学生刚刚燃起的热情; 通过教师或者学生的优秀文章来予以引 导, 学生的轻松心态就能激发出其丰富的想象。经过四个步骤的师生互动, 接下来便是学生的 写作训练了。

\section{（二） 文章操练}

教师的课堂讲解要适可而止、适量而止, 教师要起好主导作用, 关键是充分发挥学生的主 体能动性。“一言堂” 的教学模式, 无疑只能是喧宾夺主, 有百害而无一利, 不利于激发学生的 主动性。课堂如同舞台, 教师应是幕后的导演, 让学生在舞台尽情的表演, 去自由的展示自己。 因此, 教师的导入讲解后, 接下来应该让学生进行充分的文章操练。

学生的操练要有计划性, 首先是课堂中的选题构思与拟写提纲, 教师要对选题与提纲进行 适当的点评; 其次是课下的写作完善, 需要学生的精心准备。最后是作业提交与教师评析, 给 学生适当的鼓励与建设性的意见, 此环节是学生的再提高阶段。作为 “写” 的高级表现形式一 一写作, 它是书面性质的言语表达活动, 是写作者把要表达的思想内容形之于词、句、篇章的 活动。写作作品应当语言准确、结构严密、观点鲜明、信息集中。写出好的作品不但要有文字 的书写能力, 还要有安排文字 “优美化” 的能力, 是要通过 “学习” 才能实现的, 绝非轻而易 举, 一蹴而就。(《国际日报》, 2009 年 9 月 23 日) 因此, 华文写作能力的培养, 除了有条理的 写作计划安排, 还需要从三个层面对写作质量进行不断深化提高。

第一层, 锻炼书面表达, 培养华文基本写作能力。写作是一种高级思维活动。写作的心理 机制主要表现在写作的心理过程以及母语与目的语的转换。（徐子亮，2007:151）写作过程中, 需要转变学生固定的思维模式。培养学生良好的语言表达习惯, 需要重视印尼文同华文语法规 则的差异。很多学生的文章在时间表达法上明显受到了母语负迁移的影响, 如: “八点早上” 的 用法便来自印尼语的表达习惯 “jam 8 pagi / pukul 8 pagi”, 而中文的正确表达方式应该是

“早上八点”。另外, 标点符号的使用也是学生写作的一个难点。学生对标点符号的使用往往显 得束手无策, 有些甚至采取逃避的办法, 这就导致了句子过长、句式杂糅、歧义等错误, 因此, 应抽取一些典型错误重点讲评。

第二层, 真情实感的流露, 以情动人; 体味人间百态, 以理服人。如图五所示, 教学要从 学生的实际出发, 写作要贴近学生的生活。抓住学生的兴趣点, 发挥学生的爱好特长, 让学生 有话可说、有情可表。高级阶段的写作, 需要训练学生的游记、说明文、议论文、随笔、编故 事、读后感、评论、毕业论文等几种文章类型。无论哪种类型, 都必须切合印尼学生的所见所 闻所感。千岛之国的美丽风景让游记不再枯燥无味, 风云变幻中屹立千年的婆罗浮屠佛塔让说 明文妙趣横生, 雅加达的交通、治安、环境也让议论文有感可叹, 亲情、友情、爱情则会让随 笔、故事感人至深。

第三层, 艺术美感的追求, 语言的生动, 结构的合理。学生在写作中往往会有词不达意的 
困惑, 用印尼语可以写出一篇优美的文章, 而到了华文写作则显得比较生硬，有时令人费解。 本层次是写作的难点, 需要良好的华文基本功, 大量的写作训练, 丰富的词汇积累。对大多数 学生而言, 达到这一层次是比较困难的, 让学生去模仿与尝试运用文学词汇, 同时教师的不断 鼓励与示范都有助于其提高。篇章结构的设计需要给学生一些范例, 而文章的美感则需要慢慢 培养引导。

\section{（三）反馈交流}

写作是一个精细的工程, 需要不断的修改提升, 需要教师与学生的反馈交流。双向互动是 写作教学的动力之源, 有助于良好学习氛围的营造, 有助于师生情感的增进, 有助于学生自信 心的建立, 进而有助于学生写作水平的提高。如何进行高级阶段的写作教学? “Responding to student writing: Teachers need to provide helpful and informative feedback to students at a variety of points during the writing process; these feedback mechanisms will also need to vary with the teacher's purpose and the individual student's progress. Students need both informal and formal feedback from teachers, whether the feedback be assistance with brainstorming, informal feedback during class discussions, or more specific and formal feedback on written drafts.” (William Grabe, 1996: 357-358) 写作反馈需要多种渠道进行, 教师不仅在课堂上要予以点评启发, 课外的反馈尤其重要。

图 5、图 6 表明, 随着时代的发展, 网络应用越来越广泛, “网络博客” 慢慢成为一种抒 写情怀的空间, 通过 Facebook, MSN, Binusmaya, Binusblog 等网络空间, 可以和学生实时交 流。Binusmaya 是建国大学开发的一种网络教学管理程序, 教师和学生都有自己的登录名, 教 师在网站可以进行作业修改、发表信息、查看校内新闻、输入考试成绩等各种操作, 学生可以 通过网站提交作业、评价教师、查看成绩、下载教学资料等。Binusblog 则是建国大学为教师 开发的专用博客, 教师们可以进行教学交流、分享教学经验, 同时学生可以在博客里留言、发 表评论、探讨问题。另外，充分利用华文报纸，如印尼《国际日报》设有 “师生园地” ，《印 尼星洲日报》设有 “红口海” 栏目, 教师可以辅导推荐学生的文章予以发表。建国华文报是建 国大学中文系创办的华文报, 通过 “校内新闻” 可以锻炼学生的新闻写作, “学习园地” 则是 师生交流的舞台, “快乐汉语” 让学习更加轻松, “国际新闻” 则拓宽学生的视野。通过多种 渠道进行反馈, 让课外交流成为写作教学的第二课堂与有益补充。

例析: Facebook 运用——《幸福在哪里？》（马峰，《国际日报》, 2009 年 7 月 26 日)

一个慒懂的女孩, 一段忧伤的心语: “我累了, 怎么努力也无法得到幸福……幸福是什么呢? 在生活中, 遇到各种各样的困难是一定的事情。怎么能面对这种困难呢? 我总是想, 在生活中 的事情是没完没了的。我有这个力量来面对它吗? 什么时候才能愉快地过日子呢? 怎么才能得 到幸福呢? 为什么这么难? 我想, 全世界的人最需要的, 那就是幸福。幸福, 看起来是一个很 简单的事情, 但是实际上, 它是一种最难追到的事情。困难总是一直困扰着我们。我们怎么解 脱它, 它也不要离开。我必须放弃了这个小小的希望吗? ”

这段真情实感的流露, 充满了迷茫与无助。看到学生在 Facebook 博客里写的这段文章, 感 慨之余的回复: 幸福在哪里? 幸福就在我们身边! 世界不是缺少幸福, 而是缺少发现幸福的目 光! 青草的幸福是雨露的滋养, 绿树的幸福是阳光的爱抚, 碧海的幸福是游鱼的缤纷, 蓝天的 幸福是白云的可爱。你的幸福是什么呢? 幸福不代表拥有: 追求梦想的艰辛, 亲朋好友的愉悦, 青春美好的时光……这一切都充满了幸福！拥有一颗美丽、善良、充满爱的心，你就拥有了整 
个世界。一杯清茶能让一位老人陷入青春梦想的沉思, 一部佳作能让一个青年憧憬辉煌灿烂的 未来。阳光灿烂的日子, 静静的躺在海滩, 聆听大海的呼唤! 风轻拂你的面规, 撩动你的秀发, 清凉的海水四溅！这就是幸福！用心，享受生活的点滴！用爱，精心构筑理想的宏伟蓝图！用 美，追寻彼此的目光与绚烂多姿的世界!

教学反馈需要师生间的交流, 如果教师在写作教学中真正同学生互动起来, 其效果是显著 而有效的。写作, 不能只是学生的独角戏, 也需要教师发挥创作才思。教师的以身作则, 写作 示范有助于拉近与学生的距离。充分利用每一个细微处, 激发学生的写作热情。虽然例文只是 学生的牢骚之语, 但却展现了学生的写作才华。看到学生的感慨, 真情实感的抒发, 教师的及 时反馈, 不仅能够驱散学生的迷茫, 也有助于学生感悟能力的提高。其他学生可以在博客里看 到教师的回复, 相互的探讨评论将会营造出一种轻松、愉快的氛围, 让写作不再枯燥无味, 这 样师生间的交流便自然而有效。

\section{结语}

印尼华文教育有其特殊性, 曾经的教育断层对印尼华文创作界也产生了极大冲击, 目前印 尼华文写作者协会（印华作协）大多以中老年作家为主，青年作家极少，因此，印尼的华文教 育需要提高对写作教学的重视。“写作课是一门学习对汉字、词汇、语法、书写格式、标点符号 等进行综合运用的语言实践课。其教学目的是培养学生运用汉语进行书面表达, 不断纠正错误, 排除母语干扰, 逐步提高汉语书面表达能力。” (傅亿芳, 2002: 前言) 针对海外华文教学, 尤 其是印尼华文教学, 既要培养学生的基本书面表达能力, 又要重视引导学生的华文创作热情。 二者必须兼顾, 书面表达能力是语言学习的基础, 写作则是对语言的运用深化。华文写作课有 助于培养年轻一代扎实的华文基本功，也是未来印华文学延续发展、开拓新格局的希望。

\section{参考文献}

崔永华、杨寄洲 . 2002 . 汉语课堂教学技巧 [M]. 北京: 北京语言大学出版社。

蔡昌卓. 2009. : 《多维视野下的对外汉语教学研究: 第七届国际汉语教学学术研讨会论 文集》, 桂林：广西师范大学出版社。

傅亿芳. 2002. 汉语写作初阶 [M]. 北京：北京语言文化大学出版社。

纪晓静. 2002. 试论多媒体在对外汉语教学中的作用, 《外语电化教学》。

黄昆章. 2009. 困难不少，前景看好一一亲历印尼华文教育的兴衰，澳门《市民日报》，6 月 22 日。

华文教学写作课问题探析，印尼《国际日报》，2009 年 9 月 23 日。

吕必松. 1992. 华语教学讲习 [M]. 北京: 北京语言学院出版社。 
罗青松. 2002. 对外汉语写作教学研究 [M]. 北京: 中国社会科学出版社。

马峰. 2009. 秀甲天下的绿色家园一一茂物植物园游记 (Kebun Raya Bogor), 《印尼星洲日报》, 9 月 9 日。

马峰. 2009. 幸福在哪里？印尼《国际日报》，7月 26 日。

吴振邦、吕文珍. 1994. 汉语写作: 三年级用 [M]. 北京: 北京语言大学出版社。

徐子亮 . 2007. 对外汉语教学心理学 $[M]$. 上海: 华东师范大学出版社。

周振甫 . 2006. 文章例话 $[M]$. 北京: 中国青年出版社。

Gruwell, Erin. 2007. Teach with your heart: lessons I learned from the Freedom Writers, New York: Broadway Books.

Grabe, William, Kaplan, Robert B. 1996. Theory and Practice of Writing: An Applied Linguistic Perspective, London: Addison Wesley Longman limited. 